\title{
Supporting Information Bioinspired Silk Fiber Spinning System via Automated Track-drawing
}

\author{
Dave Jao ${ }^{1}$, Xiao Hu ${ }^{1,2,3}$, *Vince Beachley ${ }^{1,4}$. \\ ${ }^{1}$ Department of Biomedical Engineering, ${ }^{2}$ Department of Physics and Astronomy, and \\ ${ }^{3}$ Department of Molecular and Cellular Biosciences, \\ Rowan University, Glassboro, New Jersey 08028, United States \\ *Corresponding Author, E-mail: beachley@rowan.edu
}




\section{Supporting Information}
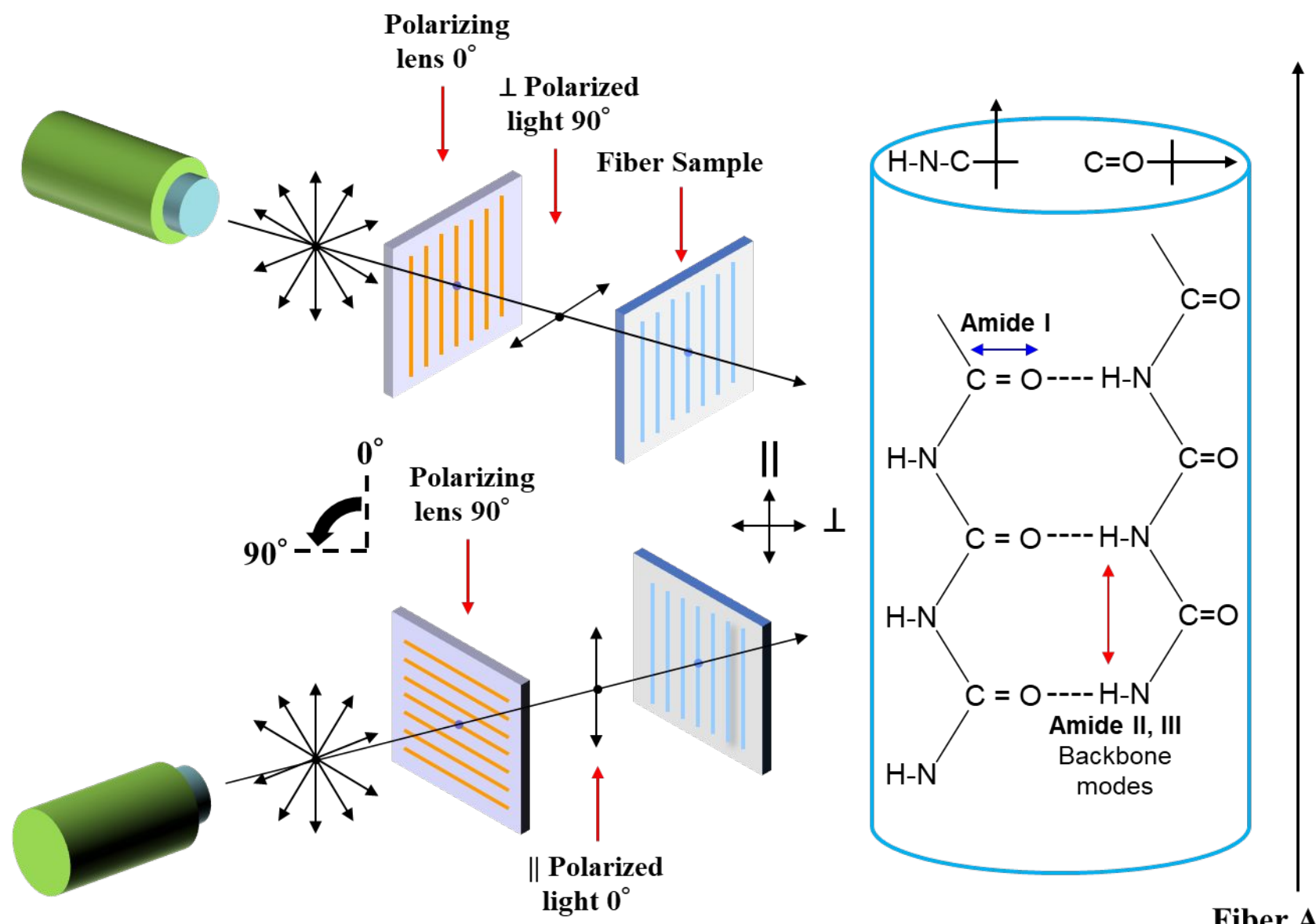

Fiber Axis

Figure S1. Fourier self-deconvolution (FSD) Amide I spectra with curve fitting for Schematic illustrating aligned SF fibers with rotating polarized FTIR used to determine the orientational behavior of $\beta$-sheets crystallites by recording the differences in $C=O$ (perpendicular to the $\beta$-sheets backbone) and $C N$ - $H$ (parallel to the $\beta$-sheets) transition moments to the $S F$ fiber axis. 
A

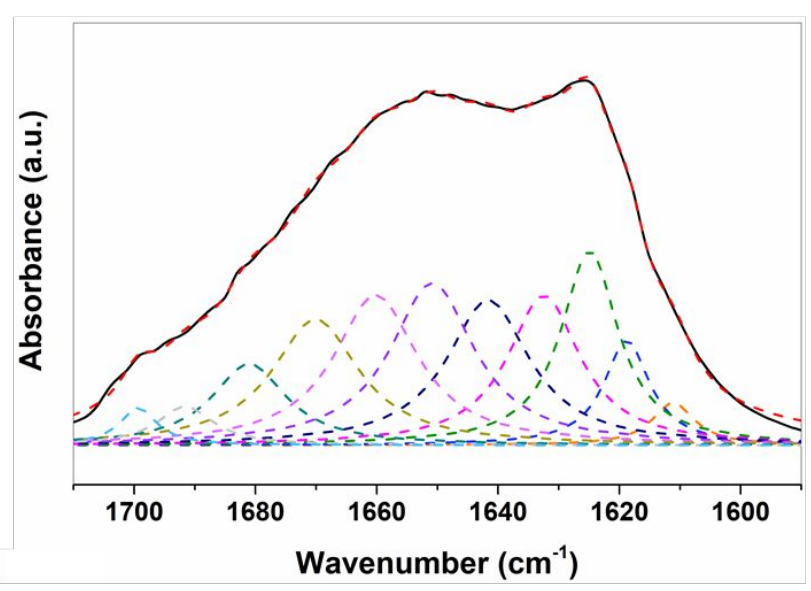

C

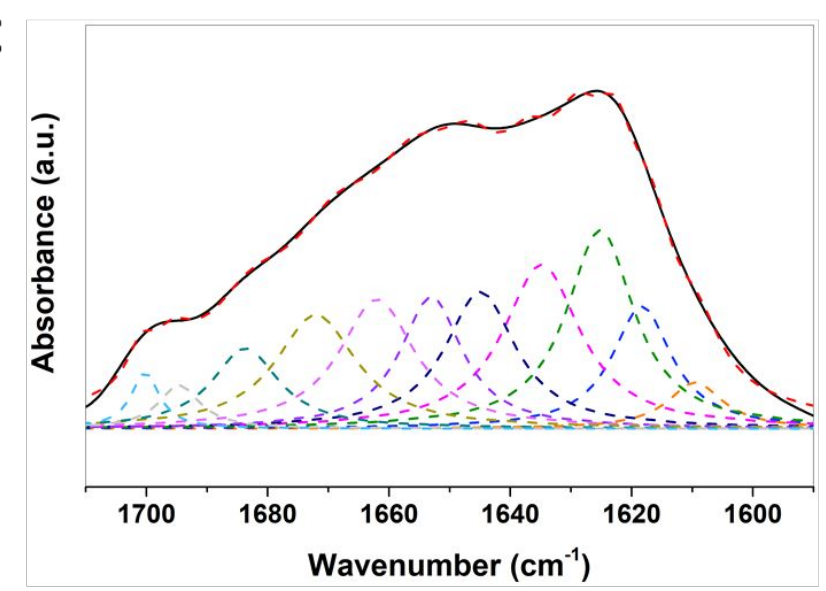

B



D

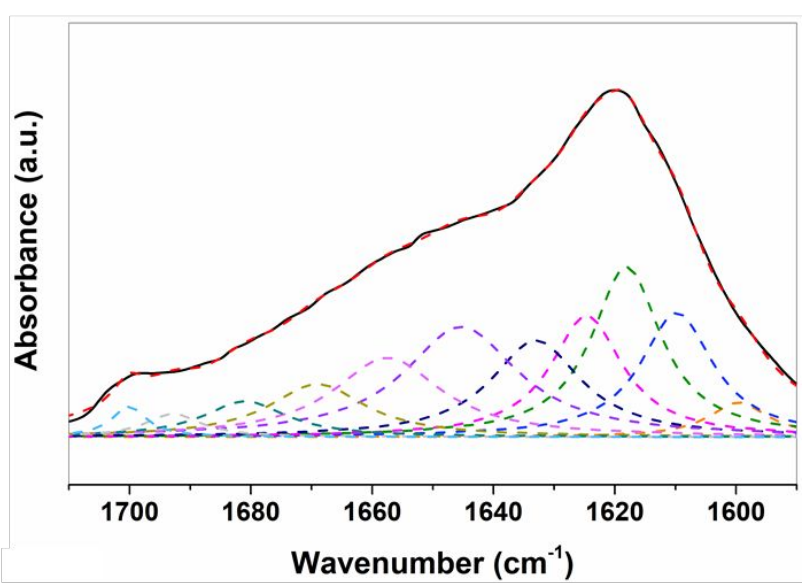

Figure S2. Fourier self-deconvolution (FSD) Amide I spectra with curve fitting for electrospun (A) Random (control), (B) DRI (undrawn), (C) DR1.5 (drawn), and (D) Film (casted) regenerated SF samples. The dashed lines represent the fitted peaks, the red dashed curve represents the summed peaks, and the black solid curve represents the original spectra. 
A

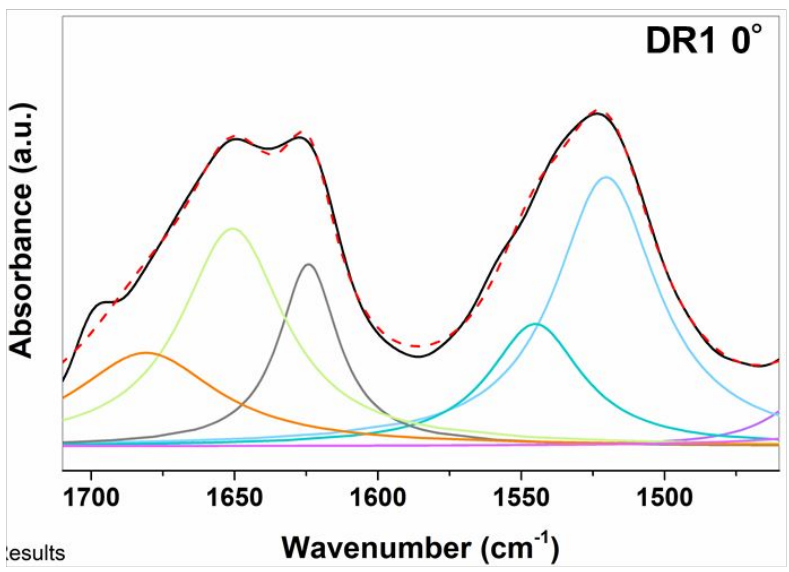

C

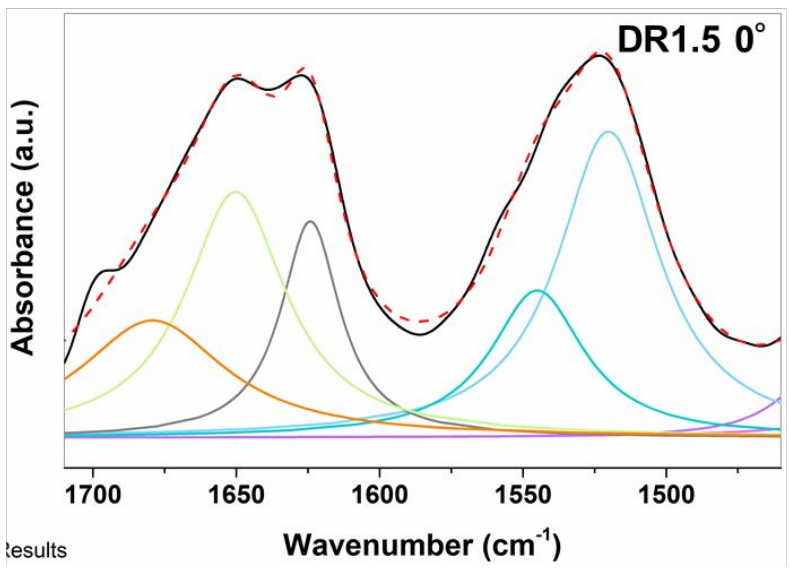

B

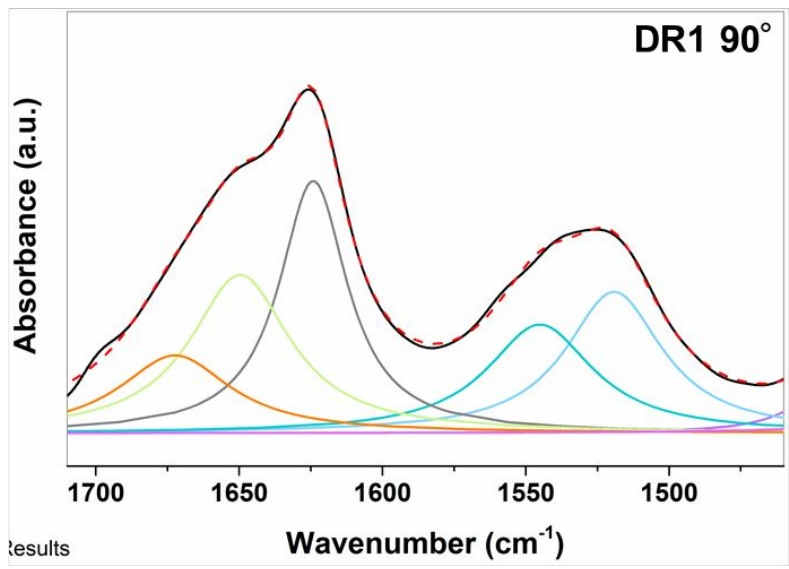

D

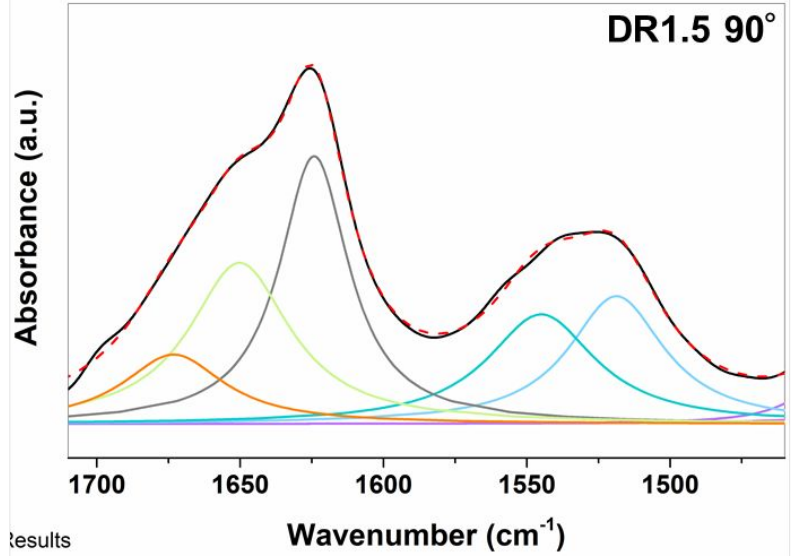

Figure S3. Joint Amide I and II curve-fitting example for the spectra deconvolution of the polarized FTIR spectra of (A-B) undrawn DRI and (C-D) post-drawn DR1.5 SF nanofibers from 1725-1425 $\mathrm{cm}^{-1}$ recorded at $0^{\circ}$ and $90^{\circ}$. The black solid curve represents the original spectra, the colored solid lines represent the fitted peaks, and the red dashed curve represents the summed peaks. 